\title{
Pharmakokinetikbasierte Gabe von Rituximab bei älteren Patienten sinnvoll
}

\author{
Neue Studiendaten weisen darauf hin, dass die pharmakokinetik- \\ basierte Applikation des Antikörpers Rituximab bei älteren \\ Patienten mit diffus großzelligem B-Zell-Lymphom (DLBCL) und \\ schlechter Prognose zu längerem Überleben führt.
}

Die Kombination aus acht Dosen Rituximab (MabThera ${ }^{\star}$ ) und sechs Zyklen CHOP (Cyclophosphamid, Doxorubicin, Vincristin, Prednison) ist Standard in der Erstlinenbehandlung bei älteren Patienten mit DLBCL. Die Therapieergebnisse könnten noch deutlich verbessert werden, wenn die Dosierung von Rituximab einem pharmakokinetischen Modell folge, wie die Daten der Phase-II-Studie SMARTE-R-CHOP-14 zeigten, berichtete Michael Pfreundschuh von den Universitätskliniken des Saarlandes in Homburg.

Im Rahmen der Studie erhielten 190 Patienten zwei Dosen Rituximab $\left(375 \mathrm{mg} / \mathrm{m}^{2}\right)$ vor Beginn der Chemotherapie und die übrigen sechs Dosen in immer größer werdenden Zeitabständen. Die Stu- dienpopulation wurde mit einer historischen Kohorte verglichen, die das konventionelle R-CHOP14-Regime erhalten hatte.

Das SMARTE-R-CHOP-14Schema führte im Vergleich zur 14-tägigen Gabe sofort zu Therapiebeginn zu höheren RituximabSerumtitern, die zudem rund 100 Tage länger erhalten blieben. Obschon die Patienten gegenüber der R-CHOP-14-Kohorte eine schlechtere Prognose aufwiesen, war das Überleben nach drei Jahren vergleichbar.

Eine Subanalyse zeigte, dass $81 \%$ der Hochrisikopatienten (International Prognostic Index [IPI] > 2) eine komplette Remission erreichten, gegenüber $68 \%$ in der Kontrollgruppe ( $p=0,035)$. Auch in Bezug auf das ereignisfreie
Überleben (67 vs. $54 \%$; p = 0,03) und das Gesamtüberleben (80 vs. $67 \% ; \mathrm{p}=0,034)$ war das SMARTER-CHOP-14- dem R-CHOP-14Schema überlegen.

Die 8 x 14-tägige Applikation von Rituximab reiche aus für Patienten mit geringer $\mathrm{Tu}$ morlast und niedrigem Risiko, so Pfreundschuh. Bei älteren Patienten mit hohem Risiko (IPI > 2) führe die pharmakokinetikbasierte Dosierung der acht Rituximab-Dosen gemäß dem SMARTE-R-CHOP-14-Protokoll hingegen $\mathrm{zu}$ wesentlich besseren Ergebnissen. „Die Ergebnisse der SMARTE-R-CHOP-14-Studie sind die besten Ergebnisse für ältere Patienten mit DLBCL, die bisher berichtet wurden", konstatierte Pfreundschuh.

ami

Pressekonferenz „ASH 2011: Neue Entwicklungen in der Therapie maligner Lymphome: Rituximab als zentraler Baustein in der Therapie des FL, CLL und des DLBBCL", 12. Januar 2012 in Frankfurt am Main; Veranstalter: Roche

\section{Prostatakarzinom \\ Knochenmetastasenfreies Überleben verlängert}

\section{Die Behandlung mit De- nosumab verzögert signifikant das Auftreten von Knochenme- tastasen bei Patienten mit Pro- statakarzinom. Dies belegen die Ergebnisse einer aktuellen Phase-III-Studie.}

Die Studie " 147 “ war eine placebokontrollierte, multizentrische Untersuchung, an der 1.432 Männer mit einem kastrationsresistenten Prostatakarzinom ohne Knochenmetastasen zu Studienbeginn teilnahmen. Sie hatten jedoch gemäß den PSA-Kriterien ein erhöhtes Risiko für die Ent- wicklung dieser Metastasen. Als Studienmedikation erhielten sie entweder Denosumab (Xgeva ${ }^{\circledR}$ ) oder Placebo [Smith AR et al. Lancet. 2012;379:39-46]. Der Einsatz von Denosumab erhöhte gegenüber der Placebogruppe das mediane knochenmetastasenfreie Überleben signifikant um über vier Monate (29,5 vs. 25,2 Monate). Dies entsprach einer Risikoreduktion von $15 \%$. Das Auftreten der ersten Knochenmetastase verzögerte sich um 3,7 Monate; das Risiko für symptomatische ossäre Metastasen sank um ein Drittel (bei- de Ergebnisse statistisch signifikant). Das Gesamtüberleben war in den beiden Studienarmen vergleichbar.

Die Autoren verzeichneten in beiden Gruppen ähnliche Raten unerwünschter Ereignisse. Unter Denosumab waren Hypokalzämie und Kieferosteonekrosen häufiger. Erstautor Smith betonte, dass die Verlängerung des medianen knochenmetastasenfreien Überlebens die Versorgung von Männern mit Prostatakarzinom verbessern könnte. red 